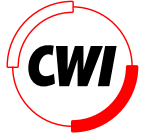

Centrum voor Wiskunde en Informatica REPORTRAPPORT

High-Concentration-Gradient Dispersion in Porous Media: Experiments, Analysis and Approximations

R.J. Schotting, H. Moser, S.M. Hassanizadeh

Modelling, Analysis and Simulation (MAS)

MAS-R9734 December 31, 1997 
Report MAS-R9734

ISSN 1386-3703

CWI

P.O. Box 94079

1090 GB Amsterdam

The Netherlands

CWI is the National Research Institute for Mathematics and Computer Science. CWI is part of the Stichting Mathematisch Centrum (SMC), the Dutch foundation for promotion of mathematics and computer science and their applications.

SMC is sponsored by the Netherlands Organization for Scientific Research (NWO). CWI is a member of ERCIM, the European Research Consortium for Informatics and Mathematics.

Copyright $(0$ Stichting Mathematisch Centrum P.O. Box 94079, 1090 GB Amsterdam (NL) Kruislaan 413, 1098 SJ Amsterdam (NL) Telephone +3120 5929333 Telefax +3120 5924199 


\title{
High-Concentration-Gradient Dispersion in Porous Media: Experiments, Analysis and Approximations
}

\author{
R.J. Schotting ${ }^{1}$ \\ CWI \\ P.O. Box 94079, 1090 GB Amsterdam, The Netherlands \\ email: ruuds@cwi.nl \\ H. Moser \\ Institut für Wasserbau und Wasserwirtschaft \\ Technischen Universität Berlin \\ Postfach 100 320, 10623 Berlin, Deutschland \\ S.M. Hassanizadeh \\ Department of Water Management, Environmental and Sanitary Engineering \\ Faculty of Civil Engineering, Delft University of Technology \\ P.O. Box 5048, 2600 GA Delft, The Netherlands \\ email: Majid.Hassanizadeh@ct.tudelft.nl
}

\begin{abstract}
Various experimental and theoretical studies have shown that Fick's law, based on the assumption of a linear relation between solute dispersive mass flux and concentration gradient, is not valid when high concentration gradients are encountered in a porous medium. The value of the macrodispersivity is found to decrease as the magnitude of the concentration gradient increases. The classical, linear theory does not provide an explanation for this phenomenon. A recently developed theory suggests a nonlinear relation between concentration gradient and dispersive mass flux, introducing a new parameter in addition to the longitudinal and transversal dispersivities. Once a unique set of relevant parameters has been determined (experimentally), the nonlinear theory provides satisfactory results, matching experimental data of column tests, over a wide range of density differences between resident and invading fluids. The lower limit of the nonlinear theory, i.e. very low (tracer) density differences, recovers the linear formulation of Fick's law.

The equations describing high concentration brine transport are a fluid mass balance, a salt mass balance in combination with a nonlinear dispersive mass flux equation, Darcy's law and an equation of state. We study the resulting set of nonlinear partial differential equations and derive explicit (exact) and semi-explicit solutions, under various assumptions. A comparison is made between mathematical solutions, numerical solutions and experimental data. The results indicate that the simple explicit solution can be used to simulate experiments in a wide range of density differences, given a unique set of experimentally determined parameters. The analysis shows that enhanced flow due to the compressibility effect, which is caused by local fluid density variations, is neglectable in all cases considered. The linear formulation of Fick's law appears to give an upperbound for magnitude of the compressibility effect.
\end{abstract}

1991 Mathematics Subject Classification: 35K65, 58G11, 76S05

Keywords and Phrases: High-concentration dispersion, Brine transport, Groundwater. Note: Work carried out under project MAS1.3 "Partial Differential Equations in Porous Media Research".

${ }^{1}$ Corresponding author 


\section{Introduction}

In recent years there has been a growing interest in the modelling of density-dependent flow and transport problems. Of particular interest have been the cases where high concentration differences in the system occur, e.g., in relation with the disposal of hazardous waste in salt formations. Some of the studies have been directed at developing numerical models capable of handling strong nonlinearities in the equations (see e.g. Leijnse [15], Kolditz et al. [14]; Oldenburg and Pruess [19]; for a list of such simulators see Kolditz et al. [14]).

Another group of studies have concentrated on the understanding of the physical processes and the validity of the basic equations used for the modelling of flow and transport. Examples are the works of Schincariol et. al. [24], Welty and Gelhar [27] and Wheatcraft [28], Kempers [12], [13] and Hassanizadeh and Leijnse [6], [5].

Commonly, salt is considered to be nonadsorbing so that the basic equations governing high concentration (HC) transport in a rigid porous medium read:

$$
n \frac{\partial \rho}{\partial t}+\operatorname{div}(\rho \mathbf{q})=0
$$

and

$$
n \frac{\partial \rho \omega}{\partial t}+\operatorname{div}(\rho \omega \mathbf{q}+\mathbf{J})=0
$$

where $n$ is porosity, $\rho$ is fluid density, $\mathbf{q}$ is the specific discharge vector (or Darcy velocity), $\mathbf{J}$ is the dispersive mass flux vector and $\omega$ is the salt mass fraction, which is defined as the salt concentration divided by the fluid density. These are equations of mass balance and need to be supplemented with equations of momentum balance. Commonly, the classical Darcy's law and a linear Fickian dispersion equation are employed for this purpose. Hassanizadeh [8], [9] suggested that these equations may not be valid when high concentration gradients exist. This was later supported by one-dimensional displacement experiments (Hassanizadeh et al. [7]) . It was found that the dispersivity does not seem to be a property of the medium but may vary from experiment to experiment; it had to be decreased as the difference in concentration of the resident and displacing fluids increased. Hassanizadeh [7] showed that this effect could not be modelled by a dependence of dispersivity on salt concentration. Instead, he proposed the following nonlinear extension of the Fickian dispersion equation

$$
\mathbf{J}(\beta J+1)=-\mathbf{D} \rho \operatorname{grad} \omega,
$$

where $\beta$ is the HC-coefficient and $\mathbf{D}$ is the well-know dispersion tensor given by:

$$
\mathbf{D}=\left(n D_{m}+\alpha_{T} q\right) \mathbf{I}+\left(\alpha_{L}-\alpha_{T}\right) \mathbf{q q} / q,
$$

Here, $D_{m}$ denotes the effective molecular diffusion coefficient (including tortuosity effects), $\alpha_{L}$ and $\alpha_{T}$ are the longitudinal and transversal dispersion lengths,,$q$ is the magnitude of the specific discharge vector and $\mathbf{I}$ is the unit tensor. Hassanizadeh and Leijnse [5] have shown that the new relationship gives very good fits to their measured breakthrough curves. Their experiments, however, were limited in number and scope. A more extensive experimental study was later carried out by Moser [17] which included the effects of varying the flow velocity. This paper reports on two different but related studies. First, the one-dimensional equations of flow and transport are analyzed mathematically. An exact and explicit solution is obtained assuming a constant flow rate in the column and the dominance of the nonlinear dispersion term in (1.3). 
A semi-explicit solution, allowing local discharge variations due to high density differences, is given. Moreover, a numerical solution of the full set of equations is provided. The three solutions are compared and conclusions are drawn with regard to the relative significance of various terms, under different concentration gradients. Second, the experimental data of Moser [17] are analyzed and the validity of the nonlinear dispersion equation (1.3) is established. It is found that the HC-coefficient varies inversely with the flow velocity $q$.

\section{Governing equations in one space dimension}

Both experimetal and analytical studies reported here deal with HC solute transport in a onedimensional domain. Therefore the governing equations presented in the previous section reduce to

$$
n \frac{\partial \rho}{\partial t}+\frac{\partial}{\partial z}(\rho q)=0
$$

where $n$ denotes the porosity of the porous medium, $\rho$ the fluid mass density, and $q=q(z, t)$ the specific discharge in the $z$-direction, and

$$
n \frac{\partial \rho \omega}{\partial t}+\frac{\partial}{\partial z}\{\rho \omega q+J\}=0
$$

where $\omega$ is the salt mass fraction and $J=J(z, t)$ the dispersive mass flux. Following Hassanizadeh \& Leijnse [5], $J$ is assumed to satisfy the nonlinear dispersion equation

$$
J(\beta|J|+1)=-D \rho \frac{\partial \omega}{\partial z},
$$

where $\beta$ is a material coefficient which may depend on the flow velocity and $D$ is the well-known one-dimensional dispersion coefficient, given by

$$
D=n D_{m}+\alpha_{L} q
$$

Neglecting pressure effects and assuming isothermal conditions, the relation between density $\rho$ and salt mass fraction $\omega$ is given by an equation of state

$$
\rho=\rho_{f} e^{\gamma \omega} .
$$

The constant $\gamma$ in (2.5) is a curve fitting constant given by $\gamma=0.6923 \approx \ln (2)$ and $\rho_{f}$ is the (reference) density of fresh water. After combining (2.1) and (2.2) (see e.g. Van Duijn et.al, 1993) and substitution of (2.5) in the result, we obtain a set of three equations in terms of $\rho, q$ and $J$ :

$$
\begin{gathered}
n \frac{\partial \rho}{\partial t}+\frac{\partial}{\partial z}(\rho q)=0 \\
n \frac{\partial \rho}{\partial t}+q \frac{\partial \rho}{\partial z}+\gamma \frac{\partial}{\partial z} J=0
\end{gathered}
$$

and

$$
J(\beta|J|+1)=-D \frac{1}{\gamma} \frac{\partial \rho}{\partial z}
$$


Next we introduce the following set of dimensionless variables:

$$
z^{*}:=z \frac{q_{0}}{D_{0}}, \quad t^{*}:=t \frac{q_{0}^{2}}{n D_{0}}, \quad q^{*}:=\frac{q}{q_{0}}, \quad J^{*}:=J \frac{1}{\rho_{f} q_{0}} \text { and } \rho:=\frac{\rho-\rho_{f}}{\rho_{s}-\rho_{f}} .
$$

The relative density difference is defined as

$$
\varepsilon=\frac{\rho_{s}-\rho_{f}}{\rho_{f}} .
$$

Moreover, we define a dimensionless parameter $\beta_{0}^{*}$ such that

$$
\beta_{0}^{*}=\beta q_{0} \rho_{f}
$$

For convenience we introduce a parameter $\theta$, defined as the ratio

$$
\theta=\frac{D}{D_{0}}
$$

Here, $D_{0}$ and $q_{0}$ denote reference parameters, defined by the conditions of the experiments. Substitution of (2.9) and (2.10) in equations (2.6)-(2.8) yields (dropping the asterisks notation for convenience)

$$
\begin{gathered}
\frac{\partial \rho}{\partial t}+\frac{\partial}{\partial z}(q \rho)+\frac{1}{\varepsilon} \frac{\partial q}{\partial z}=0 \\
\frac{\partial \rho}{\partial t}+q \frac{\partial \rho}{\partial z}+\gamma \frac{\partial J}{\partial z}=0
\end{gathered}
$$

and

$$
J\left(\beta_{0} \varepsilon|J|+1\right)=-\frac{\theta}{\gamma} \frac{\partial \rho}{\partial z} .
$$

We assume that $z$ is in the direction of decreasing density, such that $\partial \rho / \partial z<0$. This implies $J>0$ for all $(z, t) \in \mathbf{R} \times \mathbf{R}^{+}$. Under this assumption, (2.15) can be solved in terms of $\partial \rho / \partial z$, yielding

$$
J=-\frac{1}{2 \beta_{0} \varepsilon}+\frac{1}{2 \beta_{0} \varepsilon} \sqrt{1-4 \beta_{0} \varepsilon \frac{\theta}{\gamma} \frac{\partial \rho}{\partial z}}>0
$$

An implication of taking the full fluid mass balance (1.1) into account is that local density gradients cause enhanced flow in the column. During all our experiments, see Section 8, the inflow rate was kept constant. The scaled dispersion parameters $\beta_{0}$ and $\theta$ are velocity dependent. However, we disregard variations of these parameters due to the enhanced flow by assuming that $\beta_{0}$ and $\theta$ (and thereby the unscaled $\beta$ and $D$ ) only depend on the mean or background flow in the column. Under this assumption, substitution of (2.16) in (2.14) gives

$$
\frac{\partial \rho}{\partial t}+q \frac{\partial \rho}{\partial z}+\frac{\gamma}{2 \beta_{0} \varepsilon} \frac{\partial}{\partial z} \sqrt{1-4 \beta_{0} \varepsilon \frac{\theta}{\gamma} \frac{\partial \rho}{\partial z}}=0 .
$$

Equations (2.13) and (2.17) are in 'standard' form. The analysis will be confined to this set of equations, subject to initial/boundary conditions imposed by the experiments. 
As in Hassanizadeh \& Leijnse [5], we introduce the notion of a (scaled) apparent dispersion coefficient $\theta_{\text {app }}$ defined by

$$
J=-\theta_{\text {app }} \frac{\partial \rho}{\partial z} .
$$

Substitution of (2.18) in (2.16) and solving for $\theta_{\text {app }}$ yields

$$
\frac{\theta_{\text {app }}}{\theta}=\frac{2}{1+\sqrt{1-4 \beta_{0} \varepsilon \frac{\theta}{\gamma} \frac{\partial \rho}{\partial z}}} .
$$

Regardless of the choise of the reference dispersion coefficient $D_{0}$, the ratio $\theta_{\text {app }} / \theta$ is equal to the ratio $\alpha_{\text {Lappa }} / \alpha_{L}$, as defined in [5]. Notice that the apparent dispersion coefficient decreases when the gradient $-\partial \rho / \partial z$ increases for any $\beta_{0}, \varepsilon>0$. In the limit $\partial \rho / \partial z \rightarrow 0$, which is in general the case for large times or when $\varepsilon \rightarrow 0$, we obtain the well known convection-diffusion equation

$$
\frac{\partial \rho}{\partial t}+q \frac{\partial \rho}{\partial z}-\theta \frac{\partial^{2} \rho}{\partial z^{2}}=0
$$

\section{Boundary and initial conditions}

In order to keep the mathematical analysis as simple as possible we assume that the experimental column of finite length may be replaced by an infinitely long column. Effects due to the inlet boundary of a finite column usually vanish rapidly as time proceeds. This was explicitly shown by Ogata \& Banks [18] for tracer transport in finite and semi-finite columns. The experimental column is initially filled with fresh water $\left(\rho_{f}\right)$. At $t=0$, brine $\left(\rho_{s}\right)$ starts entering the column with uniform specific discharge $q_{s}$. The origin $z=0$ coincides with the position of the inlet filter of the column. This implies the following scaled initial condition for the infinite column:

$$
\rho(z, 0)=\rho_{0}(z)= \begin{cases}1 & \text { for } \quad z<0 \\ 0 & \text { for } \quad z>0\end{cases}
$$

for $z \in \mathbf{R}$, while the boundary condition for the scaled specific discharge is given by

$$
q(-\infty, t)=1 \text { for } t>0 .
$$

Subtraction of (2.14) and (2.13), and using (2.16), yields

$$
\frac{\partial q}{\partial z}=\frac{\gamma \varepsilon}{\varepsilon \rho+1} \frac{\partial J}{\partial z}=\frac{\varepsilon}{\varepsilon \rho+1} \frac{\theta}{\sqrt{1-4 \beta_{0} \varepsilon \frac{\theta}{\gamma} \frac{\partial \rho}{\partial z}}} \frac{\partial^{2} \rho}{\partial z^{2}},
$$

which indicates that (3.1) and (3.2) are sufficient conditions to obtain a unique solution. Moreover, this expression implies that density variations $(\varepsilon>0)$ affect the compressibility of the fluid, which in turn causes enhanced fluid flow. In the limit $\varepsilon \rightarrow 0$ the right hand side of (3.3) vanishes, implying $q=1$ in the whole flow domain. The limit $\beta_{0} \rightarrow 0$, i.e. constant diffusivity/dispersivity in Fick's law, has been studied in detail by Van Duijn et. al. [2], Van Duijn et. al. [3] and Van Duijn \& Schotting [4], for various flow geometries. They showed that the compressibility effect due to local density variations is in general small, and even negligible in some cases. 


\section{The explicit solution}

As a first step in our analysis, we make two approximations:

- The fluid mass balance equation (2.13) is disregarded by assuming a constant flow rate $q(z, t)=1$ for all $(z, t) \in \mathbf{R} \times \mathbf{R}^{+}$in the column. The implications of taking the full fluid mass balance equation, i.e. (2.13), into account are analyzed in the next section.

- In the nonlinear mass flux equation (2.16), the nonlinear term is assumed to dominate such that

$$
-4 \beta_{0} \varepsilon \frac{\theta}{\gamma} \frac{\partial \rho}{\partial z}>>1
$$

This assumption is justified when there exists a sharp front with a large $\varepsilon$ (which has been the case in experiments reported here).

As a result of these approximations, (2.17) reduces to

$$
\frac{\partial \rho}{\partial t}+\frac{\partial \rho}{\partial z}+\sqrt{\frac{\theta \gamma}{\beta_{0} \varepsilon}} \frac{\partial}{\partial z}\left\{-\frac{\partial \rho}{\partial z}\right\}^{\frac{1}{2}}=0 .
$$

After differentiating (4.2) with respect to $z$ and introduction of the variable

$$
w=-\frac{\partial \rho}{\partial z},
$$

we obtain

$$
\frac{\partial w}{\partial t}+\frac{\partial w}{\partial z}=\sqrt{\frac{\theta \gamma}{\beta_{0} \varepsilon}} \frac{\partial^{2}}{\partial z^{2}} w^{\frac{1}{2}}
$$

subject to the initial condition

$$
w(z, 0)=w_{0}(z)=\delta(z) \text { for } z \in \mathbf{R},
$$

where $\delta(z)$ denotes the Dirac delta function. The solution satisfies the 'mass' conservation property

$$
\int_{-\infty}^{+\infty} w(z, t) d z=\int_{-\infty}^{+\infty} w_{0}(z) d z=1 \text { for } t>0
$$

The latter follows immediately from (3.1). Because $q=1=$ constant, it is convenient to introduce a moving coordinate defined by

$$
s=z-t,
$$

and the transformation

$$
w(z, t)=v(s(z, t), t),
$$

where $v(s, t)$ is the solution of the initial value problem

$$
\frac{\partial v}{\partial t}=\sqrt{\frac{\theta \gamma}{\beta_{0} \varepsilon}} \frac{\partial^{2}}{\partial s^{2}} v^{\frac{1}{2}} \text { for }(s, t) \in \mathbf{R} \times \mathbf{R}^{+},
$$


subject to

$$
v(s, 0)=v_{0}(s)=\delta(s) \text { for } t>0 .
$$

Next we introduce a similarity variable of the form

$$
v(s, t)=t^{\alpha} f(\eta) \text { with } \eta=s t^{-\phi}
$$

Substitution of (4.11) in the conservation integral (4.6) yields

$$
t^{\alpha+\phi} \int_{-\infty}^{+\infty} f(\eta) d \eta=1 \text { for } t>0
$$

implying

$$
\alpha+\phi=0 \text { and } \int_{-\infty}^{+\infty} f(\eta) d \eta=1 .
$$

After substitution of (4.11) in (4.9), and using (4.13) in the result, we obtain

$$
\alpha=-\frac{2}{3} \text { and } \phi=+\frac{2}{3} \text {. }
$$

Thus, the function $f$ has to satisfy the boundary value problem

$$
\frac{2}{3}(\eta f)^{\prime}+\sqrt{\frac{\theta \gamma}{\beta_{0} \varepsilon}}\left(f^{\frac{1}{2}}\right)^{\prime \prime}=0 \text { for } \eta \in \mathbf{R},
$$

where the primes denote differentiation with respect to $\eta$, subject to

$$
f( \pm \infty)=0
$$

Integration of (4.15) yields

$$
\frac{2}{3} \eta f+\sqrt{\frac{\theta \gamma}{\beta_{0} \varepsilon}}\left(f^{\frac{1}{2}}\right)^{\prime}+C_{1}=0 .
$$

Then we use conditions (4.16) to obtain $C_{1}=0$. Integrating (4.17) once more gives

$$
f(\eta)=\frac{1}{\left(\frac{1}{3} \sqrt{\frac{\beta_{0} \varepsilon}{\theta \gamma}} \eta^{2}+C_{2}\right)^{2}}, \quad \text { with } C_{2}=\left(\frac{3 \pi^{2}}{4}\right)^{\frac{1}{3}}\left(\frac{\theta \gamma}{\beta_{0} \varepsilon}\right)^{\frac{1}{6}}
$$

where the value of the integration constant $C_{2}$ is determined using the conservation integral in (4.13). Back transformation to the original scaled density variable yields (skipping all details) yields

$$
\rho(z, t)=-\int_{0}^{\eta} f(\xi) d \xi+C_{3}
$$

where $C_{3}$ denotes an integration constant. Initial condition (3.1) implies: $\rho(-\infty, t)=1$ and $\rho(+\infty, t)=0$, which is used to obtain $C_{3}$. The final result, in the original scaled variables, is given by

$$
\rho(z, t)=\frac{1}{2}\left\{1-\frac{\arctan \left(\sqrt{\frac{B}{C}}(z-t) t^{-\frac{2}{3}}\right)}{C \sqrt{B C}}-\frac{(z-t) t^{-\frac{2}{3}}}{C\left(B(z-t)^{2} t^{-\frac{4}{3}}+C\right)}\right\},
$$


for $(z, t) \in \mathbf{R} \times \mathbf{R}^{+}$, where

$$
B=\frac{1}{3} \sqrt{\frac{\beta_{0} \varepsilon}{(\theta \gamma)}} \text { and } C=C_{2}=\left(\frac{3 \pi^{2}}{4}\right)^{\frac{1}{3}}\left(\frac{\theta \gamma}{\beta_{0} \varepsilon}\right)^{\frac{1}{6}}
$$

A comparison of (4.20) with experimental data will be given in Section 9 .

\section{A semi-explicit solution}

In this analysis we drop the the constant flow rate assumption. Thus we solve (2.13) and (2.17), subject to the initial/boundary conditions (3.1) and (3.2), for $\varepsilon>0$ and $\beta_{0}>0$. Assumption (4.1) is maintained. The idea is to apply a variant of the Von Mises transformation (see e.g. Von Mises \& Friedrichs [16] and Van Duijn \& Schotting [4]) in order to reduce the system (2.13), (2.17) to a single nonlinear diffusion equation. First, we introduce a new scaled density variable

$$
u=\rho+\frac{1}{\varepsilon} \text { for } \varepsilon>0
$$

Substitution of (5.1) in equations $(2.13),(2.17)$ yields

$$
\frac{\partial u}{\partial t}+\frac{\partial}{\partial z}(q u)=0
$$

and

$$
\frac{\partial u}{\partial t}+q \frac{\partial u}{\partial z}+\frac{\theta \gamma}{\beta_{0} \varepsilon} \frac{\partial}{\partial z}\left\{-\frac{\partial u}{\partial z}\right\}^{\frac{1}{2}}=0
$$

Hence, the initial/boundary conditions in the new variable are given by

$$
u(z, 0)=u_{0}(z)=\left\{\begin{array}{r}
1+\frac{1}{\varepsilon} \text { for } z<0 \\
\frac{1}{\varepsilon} \text { for } z>0
\end{array}\right.
$$

and

$$
q(-\infty, t)=1
$$

Considering the fluid balance equation (5.2) as the divergence operator in the $(t, z)$-plane, acting on a vector $(u, q u)$ we introduce a modified stream function $\Psi=\Psi(z, t)$, which satisfies

$$
u=\frac{\partial \Psi}{\partial z} \text { and } q u=-\frac{\partial \Psi}{\partial t} .
$$

The Von Mises variables are

$$
t=t \text { and } \Psi=\int_{\varsigma(t)}^{z} u(\zeta, t) d \zeta
$$

where $\varsigma(t)$ is a yet unknown function of time, which will be determined from the boundary condition on $q$, i.e. 5.5, such that $\varsigma(0)=0$. The Von Mises transformation is

$$
u(z, t)=\hat{u}(\Psi(z, t), t)=\hat{u}(\Psi, t) .
$$


Under this transformation, the system of equations (2.13) and (2.17) reduces to single nonlinear diffusion equation

$$
\frac{\partial \hat{u}}{\partial t}+\sqrt{\frac{\theta \gamma}{\beta_{0} \varepsilon}} \hat{u} \frac{\partial}{\partial \Psi}\left\{-\hat{u} \frac{\partial \hat{u}}{\partial \Psi}\right\}^{\frac{1}{2}}=0 .
$$

Multiplication of (5.9) by $\hat{u}$ and introduction of the new variable $\vartheta=\hat{u}^{2}$ yields

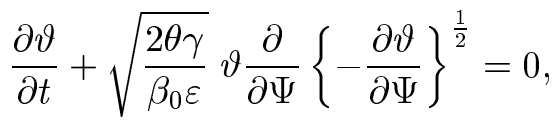

subject to

$$
\vartheta(\Psi, 0)=\vartheta_{0}(\Psi)=\left\{\begin{array}{rrr}
\left(1+\frac{1}{\varepsilon}\right)^{2} & \text { for } & \Psi<0 \\
\left(\frac{1}{\varepsilon}\right)^{2} & \text { for } & \Psi>0
\end{array}\right.
$$

Next, we look for a similarity solution of the form

$$
\vartheta(\Psi, t)=g(\eta) \text { where } \eta=\Psi t^{-\frac{2}{3}} .
$$

The function $g(\eta)$ is the solution of the boundary value problem

$$
\left\{\begin{array}{l}
-\frac{2}{3} \eta g^{\prime}+\sqrt{\frac{2 \theta \gamma}{\beta_{0} \varepsilon}}\left(\left\{-g^{\prime}\right\}^{\frac{1}{2}}\right)^{\prime}=0 \text { for } \eta \in \mathbf{R} \\
g(-\infty)=\left(1+\frac{1}{\varepsilon}\right)^{2} \text { and } g(+\infty)=\left(\frac{1}{\varepsilon}\right)^{2}
\end{array}\right.
$$

Because it is not possible to obtain an exact solution of (5.13) we have to resort to a numerical method. Since $g$ is strictly decreasing we may define the inverse of $g$ and a flux variable according to

$$
\eta=\eta(g) \text { and } h(g)=\left\{-g^{\prime}\right\}^{\frac{1}{2}}
$$

implying

$$
\eta=\frac{3}{2} \sqrt{\frac{2 \theta \gamma}{\beta_{0} \varepsilon}} g \frac{d h}{d g}
$$

and the boundary value problem

$$
\left\{\begin{array}{l}
h^{2} \frac{d}{d g}\left(g \frac{d h}{d g}\right)=\frac{2}{3} \sqrt{\frac{\beta_{0} \varepsilon}{2 \theta \gamma}} \text { for } g \in\left[\left(\frac{1}{\varepsilon}\right)^{2},\left(1+\frac{1}{\varepsilon}\right)^{2}\right] \\
h\left(\left(1+\frac{1}{\varepsilon}\right)^{2}\right)=0 \text { and } h\left(\left(\frac{1}{\varepsilon}\right)^{2}\right)=0 .
\end{array}\right.
$$

We solve this boundary value problem by discretizing the equation on a equidistant grid and solve the resulting set of nonlinear algebraic equations iteratively by means of a standard multidimensional Newton method. This yields numerical approximations of $h$ and thereby $d h / d g$. The latter is used in (5.15) to compute $g(\eta)$. Once an accurate approximation of the function $g(\eta)$ has been obtained we return to the original variables in the $(z, t)$-plane as follows. Integration of the first equation in (5.6) yields

$$
z=\int_{0}^{\psi(z, t)} \frac{1}{\hat{u}(s, t)} d s+\varsigma(t) \text { for }(z, t) \in \mathbf{R} \times \mathbf{R}^{+} .
$$


Differentiating (5.17) with respect to $t$ and using (5.6) and (5.8) in the result yields

$$
q=\varsigma^{\prime}(t)-\int_{0}^{\Psi(z, t)} \frac{\hat{u}_{t}}{\hat{u}^{2}}(\xi, t) d \xi
$$

Next we use the boundary condition (5.5) to determine the function $\varsigma(t)$, yielding

$$
\varsigma(t)=\int_{0}^{t}\left\{1-\int_{-\infty}^{0} \frac{\hat{u}_{t}}{\hat{u}^{2}}(\xi, \tau) d \xi\right\} d \tau \text { for } t>0 .
$$

Finally, after substitution of (5.19) in (5.17) and expressing $\hat{u}(\Psi, t)$ in terms of the similarity solution $g(\eta)$ we obtain

$$
\begin{gathered}
z=t^{\frac{2}{3}} \int_{0}^{\eta} \frac{1}{g(\sigma)^{\frac{1}{2}}} d \sigma+t+\frac{1}{2} t^{\frac{2}{3}} \int_{-\infty}^{0} \frac{\sigma g^{\prime}}{g^{\frac{3}{2}}} d \sigma \\
q=1+\frac{1}{3 t^{\frac{1}{3}}} \int_{-\infty}^{\eta} \frac{\sigma g^{\prime}}{g^{\frac{3}{2}}} d \sigma
\end{gathered}
$$

and

$$
u(z, t)=\hat{u}(\Psi, t)=\sqrt{g(\eta)}:=\rho(z, t)+\frac{1}{\varepsilon} .
$$

Van Duijn \& Schotting [4] showed that in the linear Fickian case, i.e. $D=\operatorname{constant}\left(\beta_{0}=0\right)$, the specific discharge distribution in an infinitely long porous column decays with $t^{-\frac{1}{2}}$, while here the specific discharge exhibits $t^{-\frac{1}{3}}$-decay.

\section{A numerical solution of the full set of equations}

Finally, for reference purposes, assumption (4.1) is also relaxed and the full set of equations (2.13)-(2.14), subject to (3.1) and (3.2), is solved numerically. This is achieved by solving the reduced equations (2.17) and (3.3) with standard schemes. The Heaviside initial condition for $u$ is approximated by a very steep error function on a fine equidistant grid. Equation (3.3) is discretized explicitly in space, providing a numerical approximation of the specific discharge distribution at that time level. A standard Crank-Nicholson scheme is used to discretize (2.17), yielding a numerical approximation of the density distribution at the new time level. The corresponding set of nonlinear algebraic equations is iteratively solved using a multi-dimensional Newton method. Once the density distribution at the new time level is known, we return to the discrete version of (3.3) to compute the specific discharge distribution at that time level, and so on.

\section{Comparison of solutions}

The explicit solution (4.20) is obtained under two simplifying assumptions, i.e. the explicit approximation (implying constant $q$ in the column) and the assumption that $-4 \beta_{0} \varepsilon \frac{\theta}{\gamma} \frac{\partial \rho}{\partial z}>>1$. The latter is the only simplifying assumption needed to obtain the semi-explicit (Von Mises) solution, which incorporates the compressibility effect due to local density variations in the fluid. 
The numerical solution is based on the full set of equations, i.e. (2.13)-(2.15), subject to (3.1) and (3.2). The compressibility effect causes local deviations from this constant (background) velocity at points where $|\partial \rho / \partial z|>0$, see e.g. (3.3) or expression (5.22), implying additional net displacement of the density front. The parameter controlling the magnitude of this effect is the relative density difference. Note that in view of (3.3), given a scaled density distribution $u(z, t)$, we may conclude $\partial q /\left.\partial z\right|_{\beta_{0}=0}>\partial q /\left.\partial z\right|_{\beta_{0}>0}$. Hence, the linear Fickian case, which corresponds to $\beta_{0}=0$, gives an upper bound for the magnitude of the enhanced flow.

At the short time scale we expect only little difference between the approximate solutions and the numerical solution. Figure 1 shows scaled density distributions at dimensionless times $t=0,0.25,0.5,0.75$ and 1.0. The parameters used to produce the graphs are: $\varepsilon=0.2, q_{0}=$ $3.209 \cdot 10^{-5} \mathrm{~m} / \mathrm{s}, \rho_{f}=1000 \mathrm{~kg} / \mathrm{m}^{3}, \theta=1, \beta_{0}=1.0 \cdot 10^{4} \mathrm{~m}^{2} / \mathrm{kg} / \mathrm{s}$, hence $\beta_{0}=\beta q_{0} \rho_{f} \approx 320$. The corresponding scaled specific discharge distributions are given in Figure 2. In case of the semi-explicit solution, the decay of the maximum of the specific discharge distribution is given by

$$
q_{\max }=1+t^{-\frac{1}{3}} G
$$

and

$$
z_{\max }=t+\frac{3}{2} t^{\frac{2}{3}} G
$$

where $G=\frac{1}{3} \int_{-\infty}^{0} \sigma g^{\prime} / g^{\frac{2}{3}} d \sigma \approx 5.7736 \cdot 10^{-2}$ for this parameter set. The additional displacement of the density front caused by the compressibility effect, is small but noticeable on the scale of Figure 1. The semi-explicit solution moves slightly faster than the corresponding numerical solution, which is due to the fact that assumption (4.1) leads to a higher apparent dispersivity in that case.

Figure 3 shows scaled density profiles for large times, i.e. $t=0,250,500,750$ and 1000 . At this time scale the semi-explicit and explicit solutions are indistinguishable. As to be expected the approximate solutions become progressively inaccurate in regions where $\partial u / \partial z$ becomes small, i.e. the top and toe regions of the density profiles. The compressibility effect is no longer noticeable. The corresponding specific discharge distributions are given in Figure 4. At these times the enhanced flow is negligible, i.e less than $1 \%$ of the scaled background flow. 


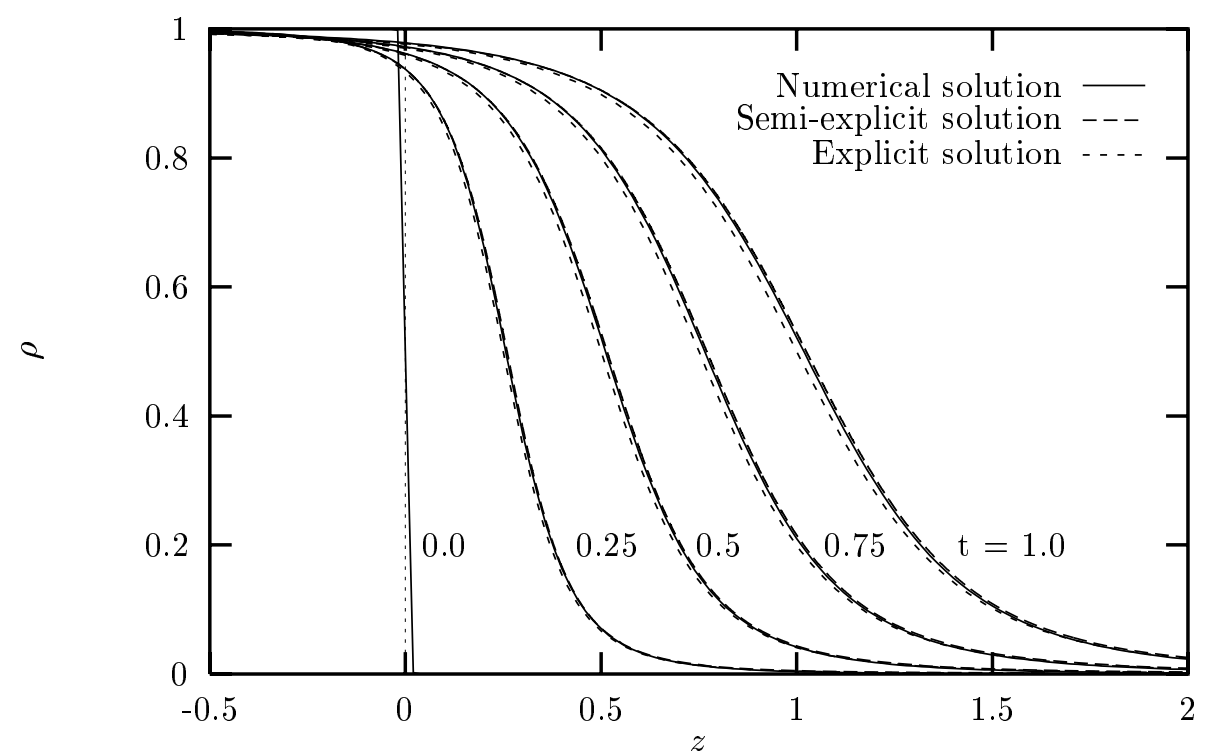

Figure 1. Comparison of solutions: density profiles at dimensionless times $t=0,0.25,0.75$ and 1.0 for $\varepsilon=0.2, \theta=1.0$ and $\beta_{0}=320$.

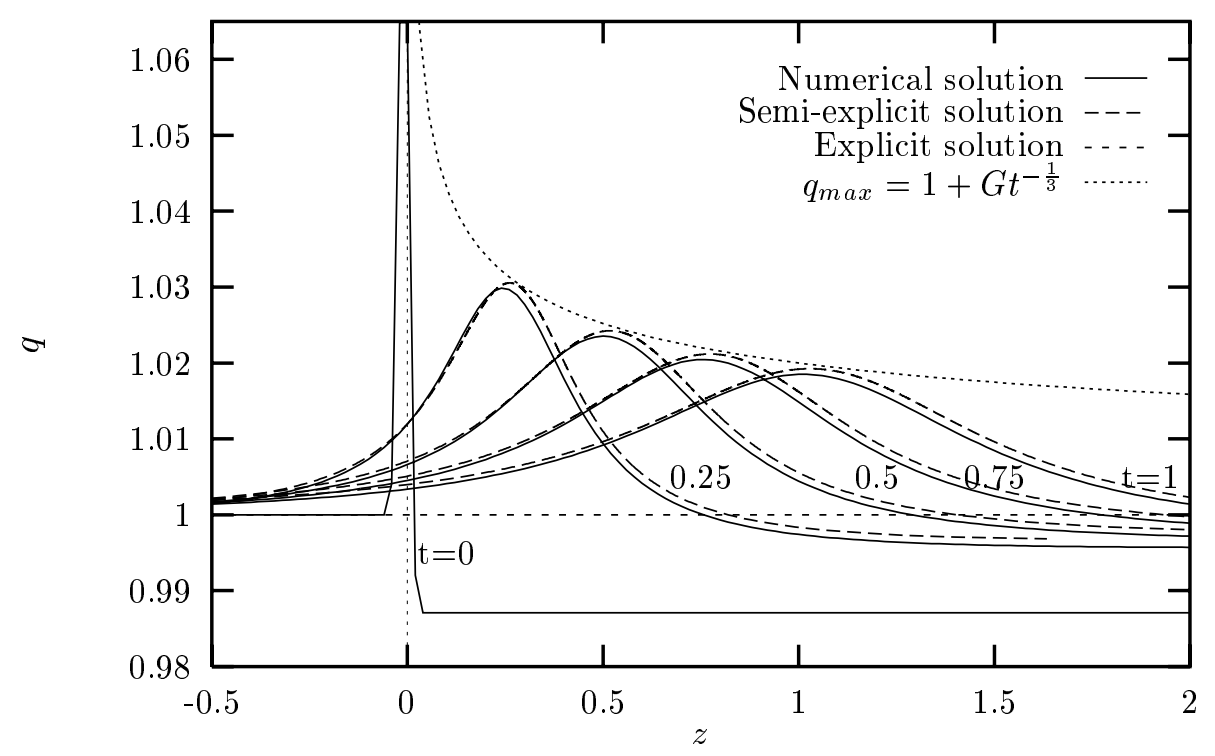

Figure 2. Comparison of solutions: specific discharge profiles at dimensionless times $\mathrm{t}=0,0.25,0.75$ and 1.0 for $\varepsilon=0.2, \theta=1.0$ and $\beta_{0}=320$. 


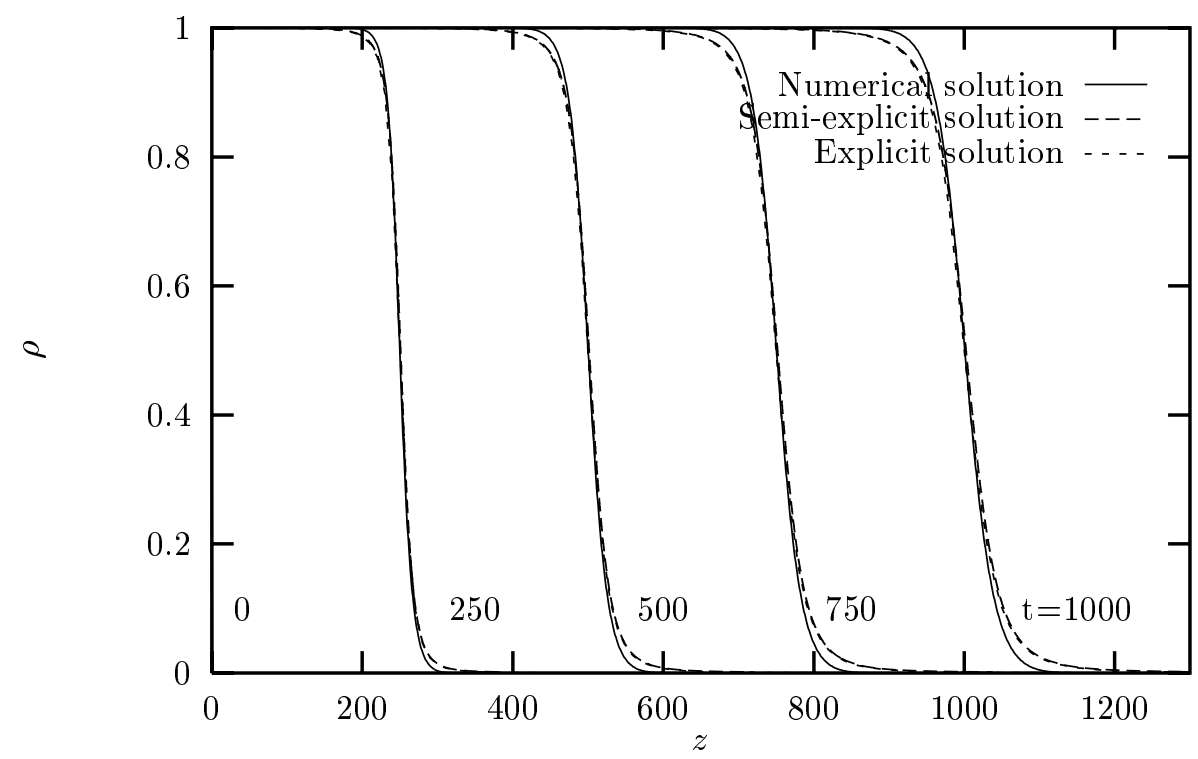

Figure 3. Comparison of solutions: density profiles at dimensionless times $\mathrm{t}=0,250,750$ and 1000 for $\varepsilon=0.2, \theta=1.0$ and $\beta_{0}=320$.

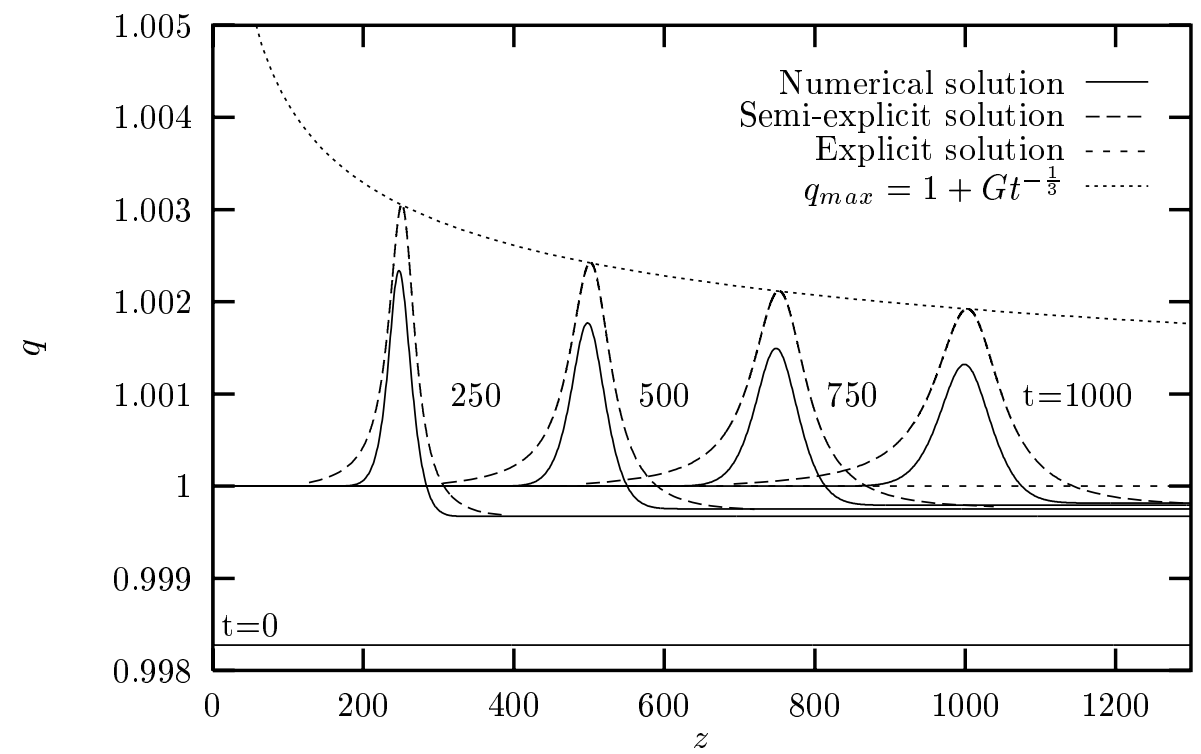

Figure 4. Comparison of solutions: specific discharge profiles at dimensionless times $t=0,250,750$ and 1000 for $\varepsilon=0.2, \theta=1.0$ and $\beta_{0}=320$.

\section{Laboratory experiments}

A series of well-controlled experiments have been performed in order to address a number of open questions related to brine transport. The experiments were aimed at determining the effects of large salt concentration differences as well as the absolute salt concentration on dispersion and to investigate the combined effect of flow velocity and large concentration differences. To this end, four series of displacement experiments were carried out (sse Table 1 through 4 for an overview):

- Fourteen tracer experiments where a low salt concentration solution was displaced by fluid with a sightly higher salt concentration (see Table 1). These are of tracer or LC-type. 
- Seven HC experiments were performed, where a low-concentration solution was displaced by a fluid with a high salt concentration (see Table 3 ). The concentration differences ranged from $2.8 \mathrm{~kg} / \mathrm{m}^{3}$ to $44.6 \mathrm{~kg} / \mathrm{m}^{3}$. The inflow rate for this series of experiments was kept to a constant value of $5.4 \cdot 10^{-4} \mathrm{~m} / \mathrm{s}$.

- Nine HC experiments were performed for which the concentration difference between the resident and displacing fluids had a constant value of $63.4 \mathrm{~kg} / \mathrm{m}^{3}$, but the flow rate was varied from $1.0 \cdot 10^{-4} \mathrm{~m} / \mathrm{s}$ to $2.3 \cdot 10^{-3} \mathrm{~m} / \mathrm{s}$ (see Table 2 ).

- Four experiments where carried out wherein a high concentration solution $\left(90 \mathrm{~kg} / \mathrm{m}^{3}\right)$ was displaced with a yet higher concentration solution (see Table 4).

Here, a brief description of the experimental setup and methods is given. For more details, the interested reader should consult Moser [17].

\subsection{The experimental setup}

The column consisted of a $1500 \mathrm{~mm}$ long plexi-glass cylinder, with an internal diameter of 206 $\mathrm{mm}$. The cylinder was filled with quartz sand with an middle particle diameter of $d_{50}=0.5$ $\mathrm{mm}$ and a uniformity coefficient of $C_{u}=d_{60} / d_{10} \approx 1.9$. A soil having a uniformity coefficient $C_{u}<2$, is considered uniform. The effective particle diameter, computed according to Beyer (see e.g. Langguth \& Voigt [11]) is $d_{e}=0.47 \mathrm{~mm}$. A sketch of the experimental setup is given in Figure 5. In order to obtain a homogeneously packed porous medium, the column

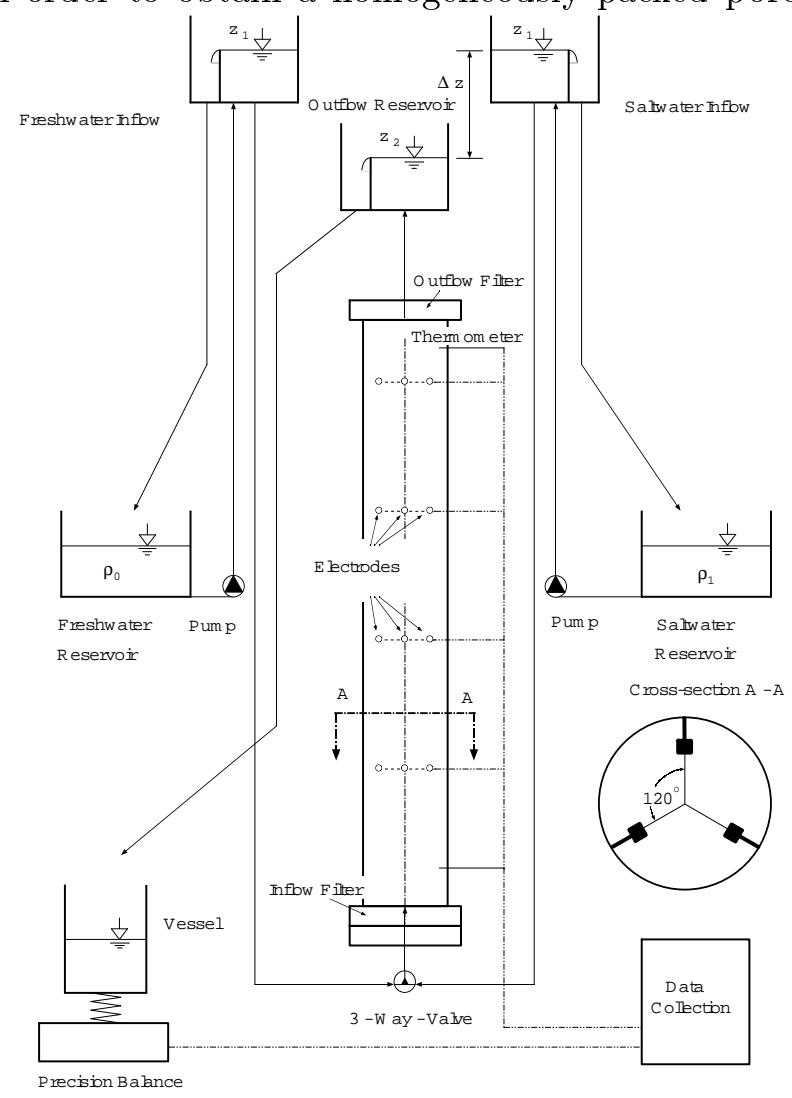

Figure 5. The experimental setup

was partially filled with water and subsequent layers (thickness $\approx 5 \mathrm{~cm}$ ) were poured into 


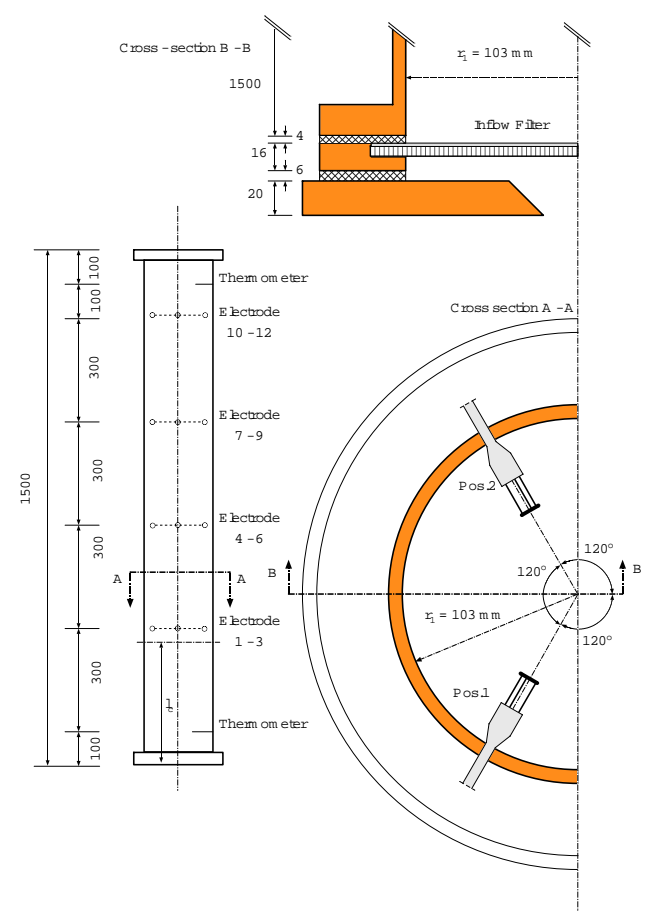

Figure 6. Details of the experimental setup

the column and each layer was packed by vibrating the column. The fluids were prepared by dissolving certain amounts of pure $\mathrm{NaCl}$ in distilled water. Both fresh and salt water circuits consisted of a storage reservoir, a pump and an overflow reservoir to ensure constant fluid pressure. The overflow reservoirs were connected to the inlet of the column by a three-way valve, which enabled instantaneous switching between the fresh and salt water circuit. The outlet of the column was also connected to an overflow reservoir whose level could be varied to create a desired pressure gradient over the porous medium. The outflowing fluid was collected in a vessel, which was mounted on an electronic precision balance. During the experiments, the amount of water in the collection vessel was recorded every 10 seconds. In case of the tracer experiments, a constant inflow rate could be obtained by fixing the distance between the water levels in the overflow reservoirs. However, when the density difference between the resident and displacing fluids was significant, the pressure gradient over the column would decease in time, causing a decrease of the flow rate. To overcome this problem, an adjustable valve was built into the salt water circuit. During high concentration experiments, this valve was gradually opened, such that the flow rate remained constant. The salt concentration was indirectly determined by measuring the electrical conductivity of the fluid in the porous medium. The electrodes consisted of three platinum/iridium wires, with a diameter of $1 \mathrm{~mm}$ and a length of $20 \mathrm{~mm}$. The electrodes protruded into the porous medium, as shown in Figure 6. Each and every electrode was calibrated in-situ. A major disadvantage of relating the electrical conductivity to the salt concentration in a fluid is the fact that the sensitivity of conductivity measurements decreases as the salt concentration increases. Experiments showed that salt concentrations of $10 \mathrm{~g} / \mathrm{kg}$ and $100 \mathrm{~g} / \mathrm{kg}$, could be reproduced within a relative error of respectively $\pm 1 \%$ and $\pm 2 \%$. All displacement experiments were carried out at constant room temperature, i.e. $20^{\circ} \mathrm{C} \pm 1^{\circ}$. The temperature of the inflow and outflow fluid was constantly monitored. 


\subsection{The LC experiments}

In the case of LG experiments, the salt mass fraction of the resident and invading fluids were, respectively, $1.0 \mathrm{~g} / \mathrm{kg}$ and $4.0 \mathrm{~g} / \mathrm{kg}$. The only quantity that was varied between the twelve tracer experiments, was the flow rate. The molecular Peclet number, defined as

$$
P_{e}=q d_{50} / D_{m}
$$

ranged from $\approx 10$ to $\approx 3000$, see Table 1 . The dispersion coefficient was determined by analyzing the breakthrough curves, measured with electrodes 10,11 and 12, i.e. at a distance of $130 \mathrm{~cm}$ from from the column inlet. electrode level in the top part of the column.

We used a least squares method to obtain an optimal fit between the analytical solution of the linear convention-diffusion equation (2.20) (constant flow rate), given by

$$
\rho=\frac{1}{2}\left(1-\operatorname{erf}\left(\frac{L-q t / n}{2 \sqrt{D t}}\right)\right)
$$

and the measured breakthrough curves. Here, $L$ denotes the position of the electrode, $q$ the specific discharge, $n$ the porosity and $D$ the fitting parameter. The porosity was determined from the measured flow rate and the breakthrough time of $c / c_{0}=0.5$. The obtained values of $D$ and $n$ are listed in Table 1. A comparison between the results of the tracer experiments and data from literature, collected by Pfannkuch [20], is given in Figure 7. The corresponding data can be found in Table 1 .

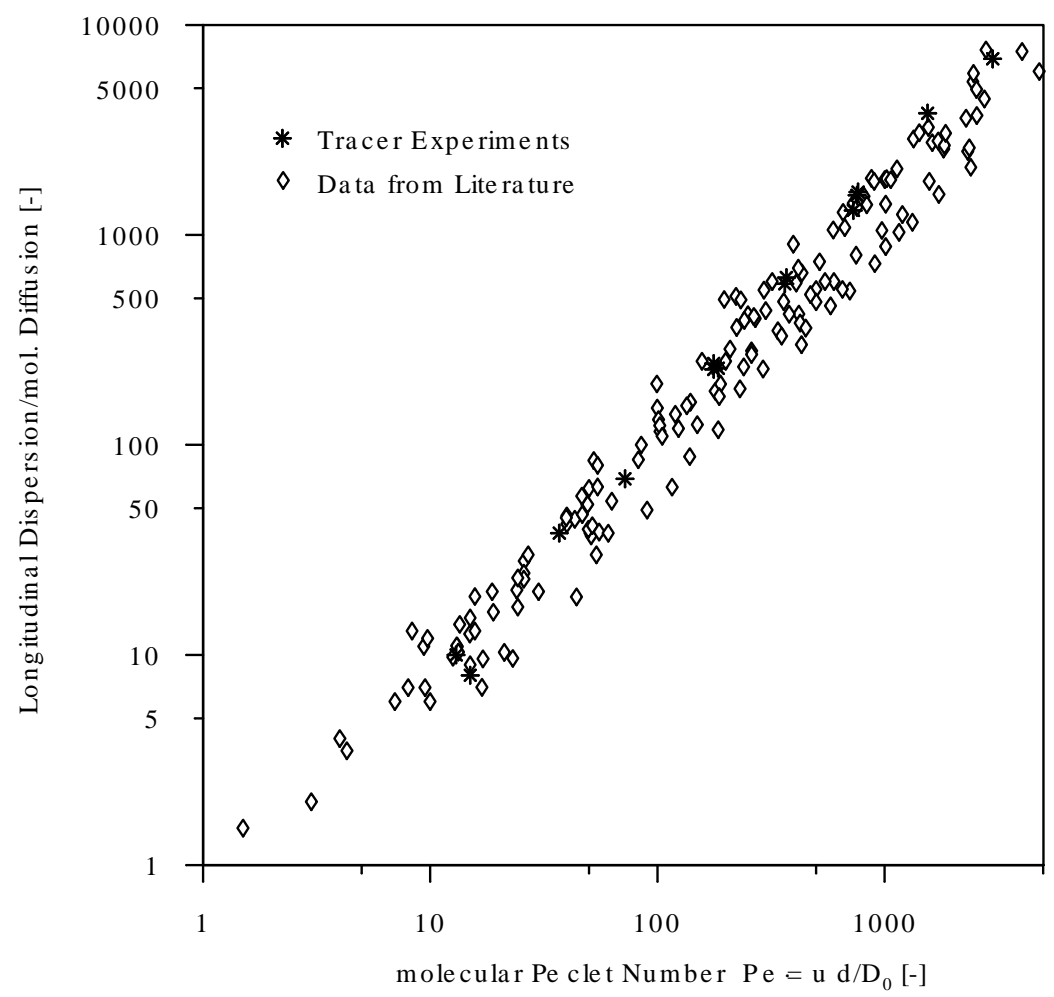

Figure 7. Comparison between data from literature and the results of the tracer experiments 


\begin{tabular}{|l|l|l|l|l|}
\hline $\begin{array}{l}\text { Experiment } \\
\text { number }\end{array}$ & Porosity & $\begin{array}{l}\text { Specific } \\
\text { discharge }[\mathrm{mm} / \mathrm{s}]\end{array}$ & $\begin{array}{l}\text { Molecular } \\
\text { Peclet numb. }\end{array}$ & $\begin{array}{l}\text { Dispersion } \\
\text { coefficient }\left[\mathrm{mm}^{2} / \mathrm{s}\right]\end{array}$ \\
\hline L10C04 & 0.382 & 0.010 & 13 & \\
L06C04 & 0.378 & 0.011 & 15 & 0.01 \\
L07C04 & 0.408 & 0.029 & 37 & 0.008 \\
L11C04 & 0.394 & 0.057 & 72 & 0.038 \\
L09C04 & 0.403 & 0.143 & 177 & 0.069 \\
L13C04 & 0.395 & 0.141 & 178 & 0.229 \\
L04C04 & 0.403 & 0.149 & 185 & 0.244 \\
L03C04 & 0.400 & 0.292 & 364 & 0.230 \\
L14C04 & 0.397 & 0.294 & 370 & 0.585 \\
L02C04 & 0.390 & 0.568 & 728 & 0.626 \\
L15C04 & 0.391 & 0.592 & 757 & 1.508 \\
L01C04 & 0.394 & 0.604 & 764 & 1.543 \\
L16C04 & 0.385 & 1.189 & 1547 & 1.600 \\
L12C04 & 0.387 & 2.312 & 2989 & 3.797 \\
\hline
\end{tabular}

Table 1. Data of the tracer experiments.

\subsection{HC experiments}

In the second set of high concentration experiments the mass fractions of the resident and displacing fluids were respectively $6 \mathrm{~g} / \mathrm{kg}$ and $100 \mathrm{~g} / \mathrm{kg}$, which amounts to $\Delta \rho=63.7 \mathrm{~kg} / \mathrm{m}^{3}$ or $\varepsilon=0.06$. The flow rate was varied such that the specific discharge ranged from $\approx 0.094$ $\mathrm{mm} / \mathrm{s}$ to $\approx 2.302 \mathrm{~mm} / \mathrm{s}$. The results are given in Table 2 . All HC experiments showed a tailing phenomenon in the high concentration region of the breakthrough curves. This is probably caused by diffusion of salt into pores where the fluid is (almost) stagnant. The apparent values of the dispersion coefficient listed in Table 2 are only indicative and obtained by fitting the error function solution (8.2) to the measured breakthrough curves. Because of the asymmetry in the breakthrough curves, due to the tailing, we only used the first part of the breakthrough curve data, i.e. up to $c / c_{0}=0.5$, to determine an apparent value of the dispersion coefficient.

\begin{tabular}{|l|l|l|}
\hline $\begin{array}{l}\text { Experiment } \\
\text { number }\end{array}$ & $\begin{array}{l}\text { Specific } \\
\text { discharge }[\mathrm{mm} / \mathrm{s}]\end{array}$ & $\begin{array}{l}\text { Dispersion } \\
\text { coefficient }\left[\mathrm{mm}^{2} / \mathrm{s}\right]\end{array}$ \\
\hline & & \\
$\mathrm{H} 09 \mathrm{C} 100$ & 2.302 & 4.153 \\
$\mathrm{H} 05 \mathrm{C} 100$ & 1.256 & 1.569 \\
$\mathrm{H} 01 \mathrm{C} 100$ & 0.653 & 0.452 \\
$\mathrm{H} 07 \mathrm{C} 100$ & 0.644 & 0.422 \\
H02C100 & 0.588 & 0.389 \\
$\mathrm{H} 10 \mathrm{C} 100$ & 0.325 & 0.189 \\
H03C100 & 0.176 & 0.068 \\
H04C100 & 0.141 & 0.069 \\
H06C100 & 0.101 & 0.036 \\
\hline
\end{tabular}

Table 2. High concentration experiments: constant density difference $\left(\Delta \rho=63.7 \mathrm{~kg} / \mathrm{m}^{3}, \mathrm{M}=\right.$ 0.91 ) and different flow rates.

In the first set of high concentration experiments, the density difference was varied from $\Delta \rho \approx 3$ $\mathrm{kg} / \mathrm{m}^{3}$ to $\Delta \rho \approx 45 \mathrm{~kg} / \mathrm{m}^{3}$, while the flow rate was fixed at a constant value, corresponding to a specific discharge of about $0.54 \mathrm{~mm} / \mathrm{s}$. 


\begin{tabular}{|l|l|l|}
\hline $\begin{array}{l}\text { Experiment } \\
\text { number }\end{array}$ & $\begin{array}{l}\text { Density } \\
\text { difference }\left[\mathrm{kg} / \mathrm{m}^{3}\right.\end{array}$ & $\begin{array}{l}\text { Dispersion } \\
\text { coefficient }\left[\mathrm{mm}^{2} / \mathrm{s}\right]\end{array}$ \\
\hline & & \\
$\mathrm{H} 01 \mathrm{C} 10$ & 2.8 & 1.535 \\
$\mathrm{H} 01 \mathrm{C} 15$ & 6.4 & 1.331 \\
$\mathrm{H} 01 \mathrm{C} 20$ & 10.0 & 1.246 \\
$\mathrm{H} 01 \mathrm{C} 25$ & 13.5 & 1.023 \\
$\mathrm{H} 01 \mathrm{C} 37$ & 21.4 & 0.987 \\
$\mathrm{H} 01 \mathrm{C} 50$ & 30.8 & 0.816 \\
$\mathrm{H} 01 \mathrm{C} 70$ & 44.6 & 0.641 \\
\hline
\end{tabular}

Table 3. High concentration experiments: constant flow rate $\left(q=5.4 \cdot 10^{-4} \mathrm{~m} / \mathrm{s}\right)$ and and various density differences.

Notice that experiments $\mathrm{H} 01 \mathrm{C} 10$ and $\mathrm{H} 01 \mathrm{C} 15$ are actually LC experiments. Table 3 clearly shows that the apparent dispersion coefficient decreases when the density difference between the fluids increases.

\subsection{High absolute concentration experiments}

The nonlinear dispersion theory indicates that the nonlinear effects depend on the fluid velocity and the magnitude of salt concentration gradients, but not on the absolute concentration levels of the resident and/or displacing fluids. To support this idea, a series of high absolute concentration experiments were carried out. The salt concentration of the resident fluid was $90 \mathrm{~g} / \mathrm{kg}$, while the concentration of the displacing fluid ranged from $100 \mathrm{~g} / \mathrm{kg}$ to $150 \mathrm{~g} / \mathrm{kg}$, see Table 4 . The specific discharge ranged from $0.45 \mathrm{~mm} / \mathrm{s}$ to $0.52 \mathrm{~mm} / \mathrm{s}$. Experiment HH01C100 $\left(\Delta \rho=7.4 \mathrm{~kg} / \mathrm{m}^{3}\right)$ can be compared with experiment $\mathrm{H} 01 \mathrm{C} 15\left(\Delta \rho=6.4 \mathrm{~kg} / \mathrm{m}^{3}\right)$ in Table 3 . Both are actually LC experiments and we notice that the dispersion coefficients have approximately the same value. The same holds for a comparison between HH01C100 and L02C04 in Table 1. These results indicate that the absolute salt concentration does not contribute to the nonlinear behavior of the dispersion coefficient.

\begin{tabular}{|l|l|l|}
\hline $\begin{array}{l}\text { Experiment } \\
\text { number }\end{array}$ & $\begin{array}{l}\text { Density } \\
\text { difference }\left[\mathrm{kg} / \mathrm{m}^{3}\right]\end{array}$ & $\begin{array}{l}\text { Dispersion } \\
\text { coefficient }\left[\mathrm{mm}^{2} / \mathrm{s}\right]\end{array}$ \\
\hline & & 1.210 \\
HH01C100 & 7.4 & 1.070 \\
HH01C110 & 13.2 & 0.980 \\
HH01C120 & 20.2 & 0.600 \\
HH01C150 & 39.4 & \\
\hline
\end{tabular}

Table 4. High absolute concentration experiments.

Moreover, we observe in Table 4 the same tendency as in Table 3: the apparent dispersion coefficient decreases as the density difference increases. Experiment H01C70 in Table 3 gives almost the same results as experiment $\mathrm{HH} 01 \mathrm{C} 150$ in Table 4, despite the fact that the salt concentration of the resident fluid in $\mathrm{HH} 01 \mathrm{C} 150$ is a factor 15 higher, when compared to the initial salt concentration in $\mathrm{H} 01 \mathrm{C} 70$.

\section{Analysis of the experimental results}

The results of the LC (tracer) experiments (Table 1) are plotted in Figure 8 in terms of the molecular Peclet number, defined in (8.1), and the ratio of the apparent longitudinal dispersion 
coefficient and the molecular diffusion coefficient. A least squares curve fitting of the LC data points, as plotted in Figure 8, yields the relation

$$
\frac{D_{L}}{D_{m}}=0.35 \cdot P_{e}^{1.26} \text { for } 10<P_{e}<3000 .
$$

Bear [1] gives a similar empirical expression which reads: $D_{L} / D_{m}=a \cdot P_{e}^{m}$, where $a \approx 0.5$ and $1<m<1.2$ in the range $6<P_{e}<200$. The latter is believed to be valid when the main spreading mechanism is caused by mechanical dispersion and transversal molecular diffusion, see [1]. In the range $200<P_{e}<10^{4}$, the mechanical dispersion is dominant, and the relation between $D_{L} / D_{m}$ and $P_{e}$ is supposed to be linear. However, when we fit the experimental data in the range $363<P_{e}<2989$ we still find some nonlinearity and the expression reads: $D_{L} / D_{m}=0.57 \cdot P_{e}^{1} .18$. This relation indicates that the dispersivity $\alpha_{L}=n D / q$ is not really a constant but increases monotonically with the specific discharge $q$. This can be indeed confirmed by the data of the LC experiments given in Table 1 . The average value of $\alpha_{L}$ is approximately $1 \mathrm{~mm}$. This is twice the average particle diameter, as to be expected for a homogeneous porous medium, see e.g. Scheidegger [23].

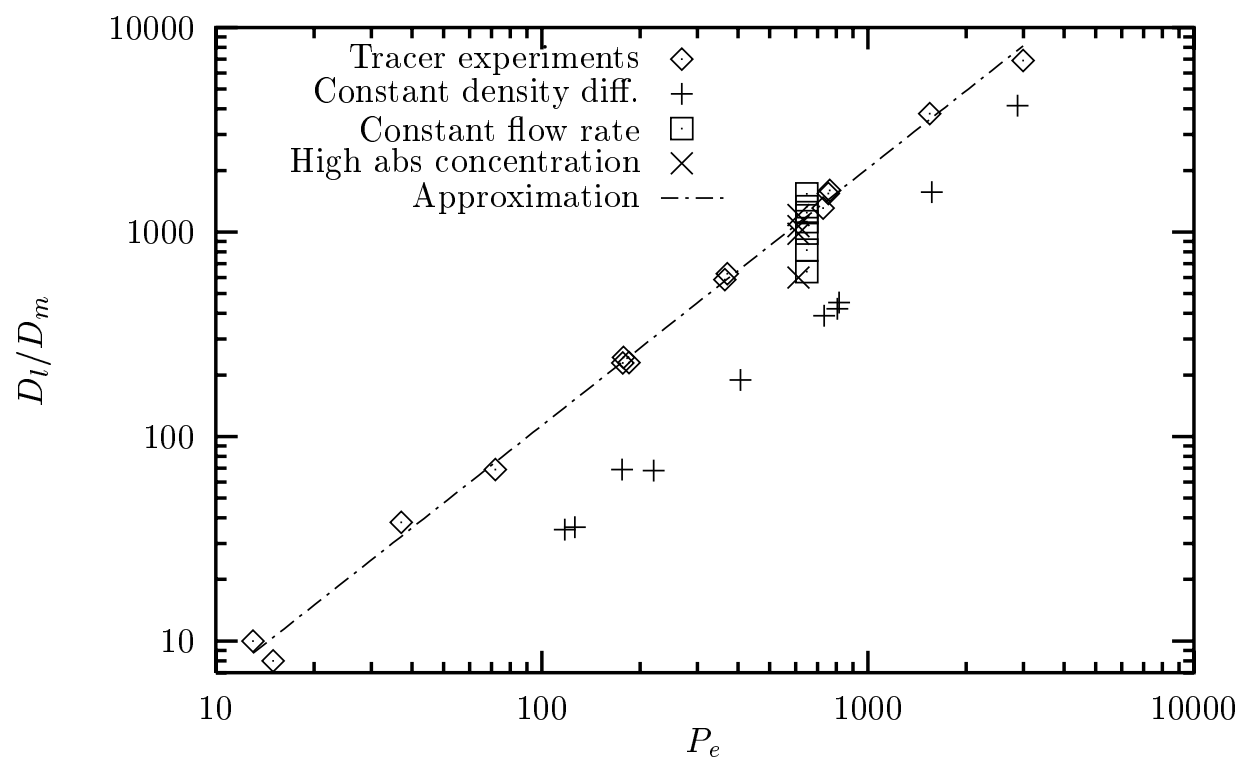

Figure 8. The ratio of the apparent dispersion coefficient and the molecular diffusion coefficient as a function of the molecular Peclet number.

Figure 8 shows the ratio of the longitudinal dispersion coefficient and the molecular diffusion coefficient as a function of the molecular Peclet number, for all experiments carried out in this study. Given a density difference of $63.7 \mathrm{~kg} / \mathrm{m}^{3}$, we observe a maximum reduction of the dispersion coefficient by a factor $\approx 3.4$. This factor decreases gradually when the molecular Peclet number (actually flow rate) increases. This behavior can be explained as follows. The porous medium is not completely homogeneous and permeability and porosity may vary locally in space, which in turn causes local velocity variations in the fluid flow. Hydrodynamic dispersion is the macroscopic outcome of these varaiations. In case of tracer density differences, the dispersion is not influenced by gravitational forces. When the density difference between the resident and displacing fluids becomes significant, the gravitational forces give rise to a reduction of the dispersion. Local horizontal density gradients cause vortices (rotation) in the fluid motion, which in turn diminishes the longitudinal spreading due to hydrodynamical dispersion in the main flow 
direction. The magnitude of the reduction of the dispersion depends upon the magnitudes of the average flow rate and the density difference.

A typical time scale associated with gravity stabilization of local velocity variations can be given by

$$
t_{g}=\frac{\alpha \mu}{\kappa g \Delta \rho}
$$

The time scale associated with the average flow in the column is

$$
t_{c}=\frac{L}{q} .
$$

If $t_{c}$ is large compared to $t_{g}$, then gravity will have enough time to reduce local velocity variations. Therefore, the ratio $t_{c} / t_{g}$ is a measure for the significance of gravity effects. In fact, this ratio is identical to the Rayleigh number

$$
R_{a}=\frac{t_{c}}{t_{g}}=\frac{\kappa g \Delta \rho L}{\mu \alpha_{L} q}
$$

When using the Kozeny-Carman equation (see Bear [1]) to compute the intrinsic permeability of the porous medium, we obtain $\kappa \approx 2.0 \cdot 10^{-10} \mathrm{~m}^{2}$. In case of the constant density difference experiments, the Rayleigh number ranges from $R_{a}=1640\left(P_{e} \approx 100\right)$ to $R_{a}=72\left(P_{e} \approx 3000\right)$. Here we used $\mu=1.0 \cdot 10^{-3} \mathrm{~kg} / \mathrm{ms}, \Delta \rho=63.7 \mathrm{~kg} / \mathrm{m}^{3}, L=1.3 \mathrm{~m}, \alpha_{L}=1.0 \cdot 10^{-3} \mathrm{~m}$ and $\kappa=2.0 \cdot 10^{-10}$. Figure 8 shows indeed a decrease of the difference between the tracer dispersion coefficient and the HC dispersion coefficient for high Peclet numbers.

In any case, Figure 8 shows that dispersion coefficient as found in the classical Fickian equation is a nonlinear function of flow velocity and salt concentration differences. The nonlinear dispersion theory proposed in [5] is believed to account correctly for the density effects. Indeed, the experiments reported in [6], although limited in number and scope, give evidence that, for a certain flow rate, it is possible to determine a single value the parameter $\beta$, such that breakthrough curves of experiments with concentration differences ranging from $1.51 \mathrm{~g} / \mathrm{kg}$ (tracer concentrations) to $232.43 \mathrm{~g} / \mathrm{kg}$ can be simulated with a reasonable accuracy.

We computed an optimal value of $\beta$ from the constant flow rate experiments, listed in Table 3 , by fitting both the explicit solution and the full numerical solution to breakthrough curves. The results for $q=5.4 \cdot 10^{-4} \mathrm{~m} / \mathrm{s}$ are listed in Table 5 . As to be expected, we obtain an almost constant value of $\beta$ by fitting the numerical solution to the experimental data. The $\beta$-values, obtained with the explicit solution become progressively inaccurate for decreasing density differences. Both results are shown in Figure 9. When the displacing fluid is fresh water $\left(\rho_{f} \approx 1000 \mathrm{~kg} / \mathrm{m}^{3}\right)$, the explicit solution appears to be accurate for the range $\rho_{s} \approx 1025 \mathrm{~kg} / \mathrm{m}^{3}$ (sea water) up to $\rho_{s} \approx 1300 \mathrm{~kg} / \mathrm{m}^{3}$ (the saturation limit of $\mathrm{NaCl}$ in water). This emphasizes the applicabillity of the explicit solution, at least for this constant flow rate data set. A more general conclusion with respect to the validity of the explicit solution cannot be gained from the experimental results presented in this study. 


\begin{tabular}{|l|l|l|l|l|}
\hline $\begin{array}{l}\text { Experiment } \\
\text { number }\end{array}$ & $\begin{array}{l}\text { density } \\
\text { difference }\left[\mathrm{kg} / \mathrm{m}^{3}\right]\end{array}$ & $\begin{array}{l}\text { Dispersion } \\
\text { coefficient }\left[\mathrm{mm}^{2} / \mathrm{s}\right]\end{array}$ & $\begin{array}{l}\beta_{\text {num }} \\
{\left[\mathrm{s} \mathrm{m}^{2} / \mathrm{kg}\right]}\end{array}$ & $\begin{array}{l}\beta_{\text {expl }} \\
{\left[\mathrm{s} \mathrm{m}^{2} / \mathrm{kg}\right]}\end{array}$ \\
\hline & & 0.641 & 6228 & 6240 \\
H01C70 & 44.6 & 0.818 & 6013 & 6101 \\
H01C50 & 30.8 & 0.987 & 6490 & 6749 \\
H01C37 & 21.4 & 1.023 & 6447 & 7133 \\
H01C25 & 13.5 & 1.246 & 6296 & 8333 \\
H01C20 & 10.0 & 1.331 & 6366 & 11574 \\
H01C15 & 6.4 & 1.535 & 6283 & 19841 \\
H01C10 & 2.8 & & 6303 & - \\
\hline$\beta_{\text {average }}$ & & \multicolumn{3}{|l}{} \\
\hline
\end{tabular}

Table 5. Obtained $\beta$-values from the constant flow rate experiments $\left(q=5.4 \cdot 10^{-4} \mathrm{~m} / \mathrm{s}\right)$

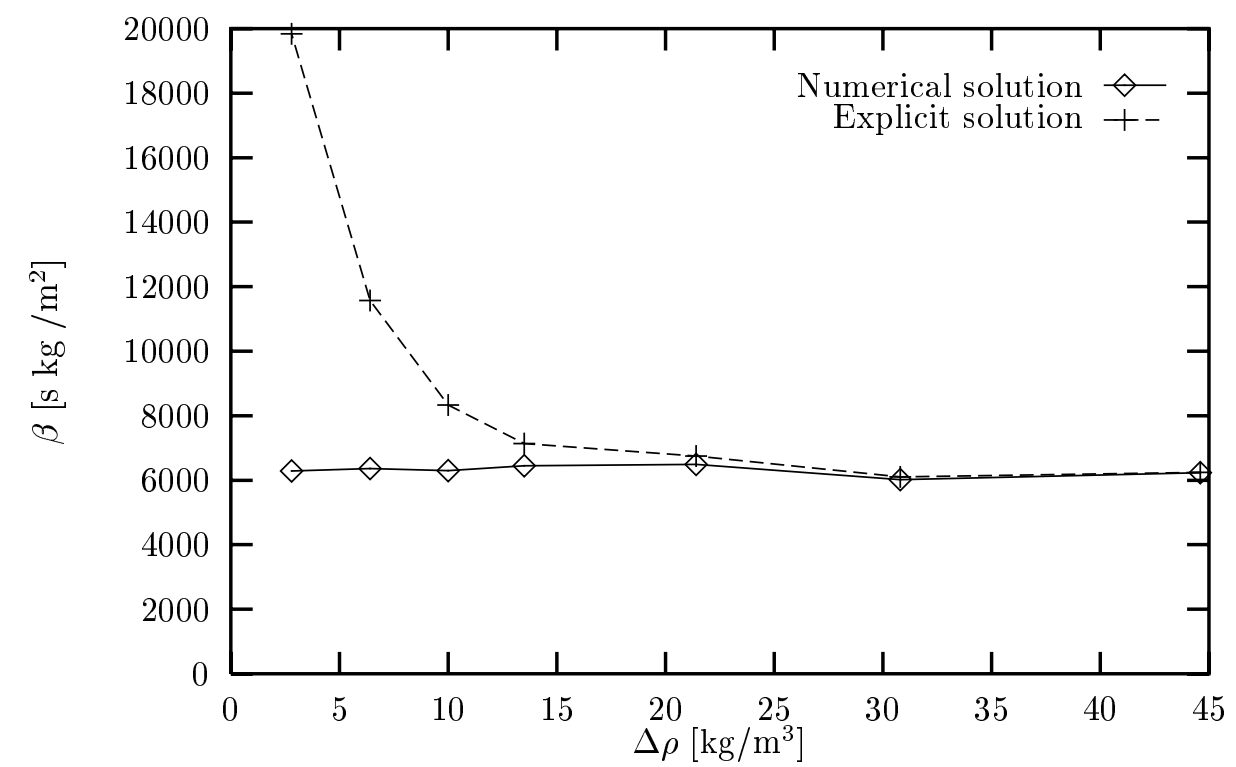

Figure 9 Comparison of $\beta$-values obtained by fitting the numerical and explicit solution to the breakthrough curves of the constant flow rate experiments

The breakthrough curves corresponding to the constant density difference experiments listed in Table 2 , were analyzed in a similar fashion. The obtained $\beta$-values are plotted as a function of the specific discharge on a log-log scale in Figure 10. A least squares curve fitting yields an approximate expression for $\beta(q)$, given by

$$
\beta(q)=\frac{0.0125}{q^{1.76}} \text { for } 9.0 \cdot 10^{-5}<q<3.0 \cdot 10^{-3} \mathrm{~m} / \mathrm{s} .
$$

This indicates that the nonlinear effect decreases with increasing flow rate. This is in correspondence with our discussion of the Rayleigh number and the balance between gravitational and convective transport. Substitution of (9.7), (2.4) (disregarding molecular diffusion) in equation (2.8), and solving for $J$ yields

$$
J=\frac{q^{n}}{2 A}\left\{\sqrt{1-4 \alpha_{l} A \gamma^{-1} q^{1-n} \frac{\partial \rho}{\partial z}}-1\right\},
$$

where $A=0.0125$ and $n=1.76$. This is the one-dimensional dispersive mass flux equation in terms of the original variables. 


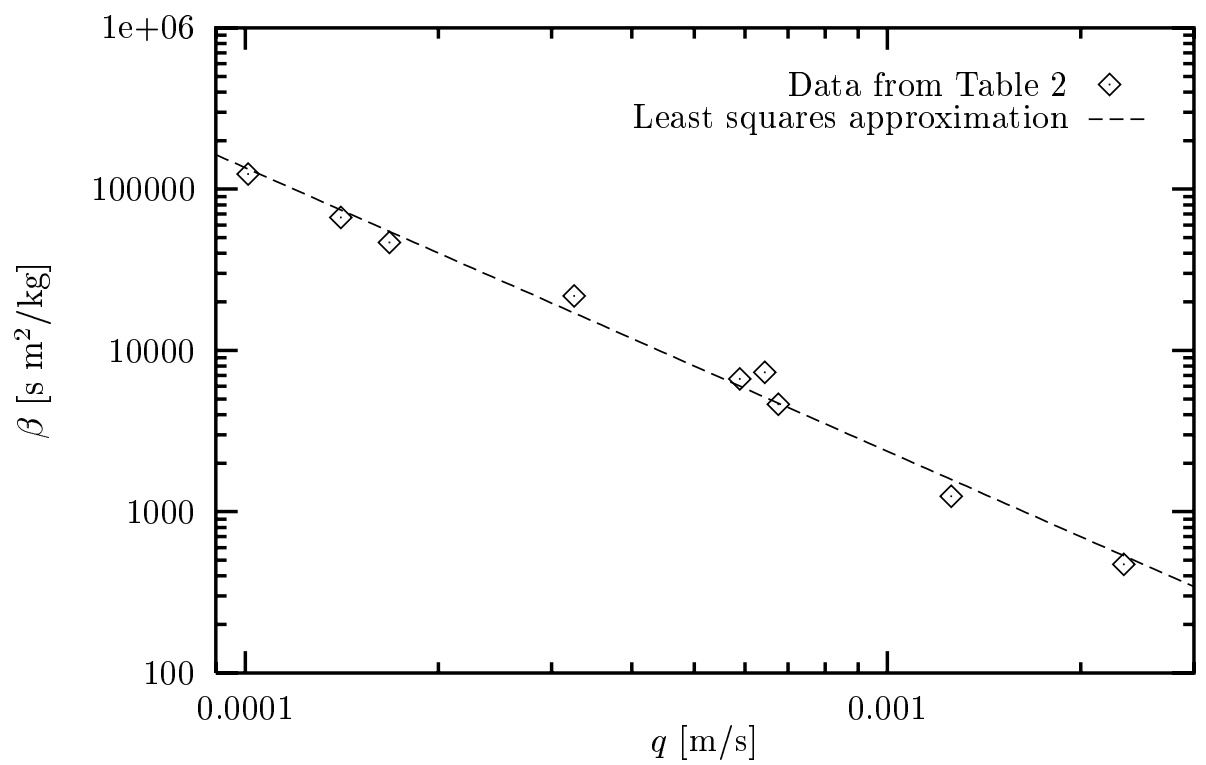

Figure 10. The parameter $\beta$ as a function of $q$ for the constant density difference experiments

\section{Discussion}

The experimental results presented in this study, confirm the validity of the nonlinear dispersive mass flux equation (2.3) and provide an empirical relationship for the $\mathrm{HC}$ dispersion coefficients in terms of the specific discharge. In the $\mathrm{HC}$ experiments, the specific discharge ranged from $q=9.0 \cdot 10^{-5} \mathrm{~m} / \mathrm{s}$ to $3.0 \cdot 10^{-3} \mathrm{~m} / \mathrm{s}$. These are relatively high when compared to groundwater discharges in the field, typically ranging from $3.0 \cdot 10^{-7} \mathrm{~m} / \mathrm{s}(0.03 \mathrm{~m} /$ day $)$ to $3.0 \cdot 10^{-5} \mathrm{~m} / \mathrm{s}(3.0$ $\mathrm{m} /$ day).

When we extrapolate expression (9.7) for $q>3.0 \cdot 10^{-3} \mathrm{~m} / \mathrm{s}$, neglecting any high velocity effects, and pass to the (theoretical) limit $q \rightarrow+\infty$ we obtain $\beta(+\infty) \rightarrow 0$. This implies that the dispersive mass flux equation (2.3) reduces to Fick's law and HC experiments can be simulated using the tracer equations and the tracer dispersion coefficient. Extrapolation of (9.7) for $q<9.0 \cdot 10^{-5} \mathrm{~m} / \mathrm{s}$, and passing to the limit $q \rightarrow 0$ yields $\beta(0) \rightarrow \infty$. This indicates that for low discharges (high Rayleigh numbers) the gravity effect, i.e. the nonlinear term in (1.3), completely dominates the dispersive behavior. From the physical point of view, both discharge limits are realistic in a qualitative sense. Additional experiments should be carried out to gain information about the behavior of $\beta(q)$ for discharges $q<9.0 \cdot 10^{-5} \mathrm{~m} / \mathrm{s}$ and $q>3.0 \cdot 10^{-3}$ $\mathrm{m} / \mathrm{s}$. A particular interesting limit to study is the transition from pure diffusive transport to dispersion dominated transport under $\mathrm{HC}$ conditions.

Substitution of (9.8) in (2.1) and (2.2) yields a set of equations that no longer allows a Von Mises transformation. In fact, application of the Von Mises transformation is restricted to those cases where the longitudinal tracer dispersion coefficient $D$ is constant, i.e. not a function of the local specific discharge, see [4]. A constant tracer dispersion coefficient may account for heterogeneities of the porous medium in an averaged sense, for instance by setting $D=\alpha_{L} q_{0}$, where $q_{0}$ denotes a background or mean specific discharge.

When using the latter, we can quantify the magnitude of the volume or compressibility effect in the experiments. To this end, we use the data of HC experiment H09C100 (see Table 3 ) and expressions (7.24) and (7.25). The breakthrough time of the 50\%-level of the density 
in experiment $\mathrm{H} 09 \mathrm{C} 100$ is $t_{50 \%}=220 \mathrm{~s}$. The dimensionless breakthrough time is given by $t_{50 \%}^{*}=423$, see the scaling rules (2.9). Tracer experiment L12C04 was used to obtain values for $D$ and $n$. At $t_{50 \%}$, the relative magnitude of the maximum of the specific discharge with respect to the back ground flow $q_{0}$ can be determined from expression (7.24). This yields $\left(q_{\max }-q_{0}\right) / q_{0} \approx 0.8 \%$. The additional relative displacement of the density front at $t_{50 \%}$, given by $(7.25)$, amounts to $\approx 1.2 \%$. Both results indicate that volume effects can be disregarded. Such variations are not noticeable within the accuracy of the experiments.

The constant flow rate experiments show a significant reduction of the dispersion coefficient in case of an increasing density difference between the resident and displacing fluids. The tracer dispersion coefficient has to be lowered by a factor $\approx 2.4\left(\mathrm{H} 01 \mathrm{C} 70, \Delta \rho=44.6 \mathrm{~kg} / \mathrm{m}^{3}\right)$ in order to obtain a reasonable fit between the solution of the classical equations and the experimental data. Experiment H02C100, with approximately the same flow rate $\left(5.88 \cdot 10^{-4} \mathrm{~m} / \mathrm{s}\right.$ instead of $5.4 \cdot 10^{-4} \mathrm{~m} / \mathrm{s}$ ) and density difference $63.7 \mathrm{~kg} / \mathrm{m}^{3}$, exhibits a reduction by a factor $\approx 4$. Figure 11 shows the scaled dispersion coefficient for the constant flow rate experiments (including $\mathrm{H} 02 \mathrm{C} 100)$ as a function of the density difference. The corresponding least squares approximation is given by $D_{L} / D_{0}=1.0158 e^{-0.0212 \Delta \rho}$, where $D_{0}=1.535 \cdot 10-6 \mathrm{~m}^{2} / \mathrm{s}$.

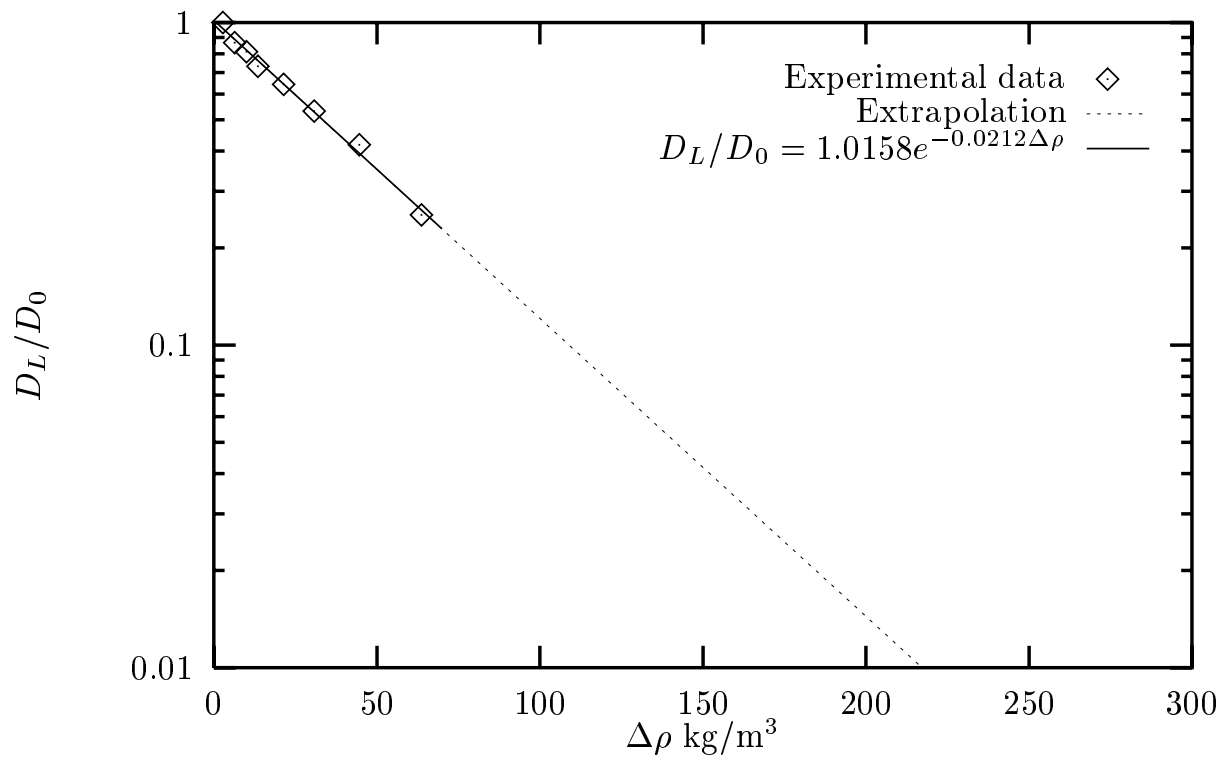

Figure 11. Scaled longitudinal dispersion coefficient as a function of the density difference for the constant flow rate experiments.

Extrapolation of the least squares approximation yields a reduction of the tracer dispersion coefficient by a factor $>100$ for almost saturated brines. Whether this is extrapolation is realistic in the physical sense has to be determined experimentally in a future study.

An other question that needs to be addressed concerns the physical phenomenon that is assumed to cause the reduction of the apparent dispersion coefficient in HC transport of brines. Is the explanation that local horizontal density gradients at pore scale induce fluid rotation (vortices) such that the dispersion of salt is reduced when compared to the tracer dispersion correct? The latter is very likely, but cannot be proved at this time. Also, simple conceptual models for $\mathrm{HC}$ transport, gaining insight in the process and its governing equations, are still lacking. For tracer dispersion, such models, have proven to be very useful, see e.g. Taylor [25], [26], De Josselin De Jong [10], Saffman [21], [22].

If gravity is indeed responsible for the observed dispersion reduction in $\mathrm{HC}$ transport, then it is of fundamental importance to find the relation between the proposed nonlinear flux equation (2.3) and the induced flow due to local horizontal density gradients. The derivation of (2.3) in 
[5] is formally correct, but is not directly linked to the physical phenomenon that causes the HC dispersion effect. Such a relation might be established by averaging the governing pore scale equations and/or by homogenization techniques. We leave these questions for a future study.

\section{Conclusions}

- This study concerns the mathematical and experimental analysis of brine dispersion in a saturated porous medium. Both low and high concentration experiments have been carried out. The LC (tracer) experiments presented in this paper are in excellent agreement with LC experiments reported in literature.

- The experiments show that for low concentration differences (even if the absolute salt concentration is high), the linear Fickian theory models the observed dispersion satisfactorily. In case of high concentration differences, the Fickian theory fails.

- All experiments reported here, both LC and HC, confirm the validity of the nonlinear HC dispersion theory given in [5].

- High absolute concentration experiments show that the HC dispersion coefficient is independent of the absolute salt concentration of fluids.

- The HC dispersion coefficient $\beta$ is found to be independent of the salt concentration differences or gradients. It depends only on the flow rate. A relation between $\beta$ and the specific discharge is established.

- An explicit solution for HC transport (assuming dominance of the nonlinearity in the salt flux equation and constant flow rate in the column) is given. This approximate solution can be used to simulate HC experiments in a wide range of density differences.

- A semi-explicit solution of the full set of governing equations (again assuming dominance of the nonlinearity in the salt flux equation) is obtained. Fluid volume changes due to high concentration gradients are taken into account. The results are used to quantify the volume effects in the $\mathrm{HC}$ experiments. It turns out that these effects can be disregarded for all experiments considered.

- The constant density HC experiments show a decrease of the difference between the tracer and the HC dispersion coefficients for increasing flow rates. This indicates that local (pore scale) horizontal density gradients might be responsible for the nonlinear $\mathrm{HC}$ dispersion effect. At high flow rates (large Peclet numbers) local gravity driven (stabilizing) flows become relatively un important (low Rayleigh numbers).

\section{References}

[1] Bear,J, Dynamics of fluids in porous media, Elsevier, New York, 1972.

[2] Van Duijn, C.J. , Peletier, L.A. \& Schotting, R.J., On the analysis of brine transport in porous media, Euro. Jnl. of Appl. Mathematics, Vol 4 (1993), pp. 271-302.

[3] Van Duijn, C.J. , Peletier, L.A. \& Schotting, R.J., Brine transport in porous media: selfsimilar solutions, CWI Report AM-R9616, November 1996. 
[4] Van Duijn, C.J. \& Schotting, R.J. , On the use of Von Mises transformation in brine transport in porous media, CWI Report in preparation 1997.

[5] Hassanizadeh, S.M. \& Leijnse, Anton, A non-linerar theory of high-concentration-gradient dispersion in porous media, Adv. Water Resour. ,Vol. 18 (1995), pp. 203-215

[6] Hassanizadeh, S.M. \& Leijnse, A., On the modelling of brine transport in porous media, Water Resour. Res., Vol. 24 (1988), pp. 321-330

[7] Hassanizadeh, S.M., Leijnse, A., De Vries, W.J. \& Stapper, R.A.M., Experimental study of brine transport in porous media, Report 728514005, RIVM, Bilthoven, The Netherlands, 1990.

[8] Hassanizadeh, S.M., Derivation of basic equations of mass transport in porous media. Part 1. Macroscopic balance laws, Adv. Water Resour. 9 (1986), p.p. 196-206

[9] Hassanizadeh, S.M., Derivation of basic equations of mass transpo rt in porous media. Part 2. Generalized Darcy's and Fick's laws, Adv. Water Resour. 9 (1986), p.p. 207-222.

[10] De Josselin De Jong, G., Longitudinal and transverse diffusion in granular deposits, Trans. Amer. Geophys. Union 39 (1958), p.p. 67-74.

[11] Langguth, H.R. \& Voigt, R., Hydrologische Methoden, Springer Verlag, Berlin (1980).

[12] Kempers, L.J.T.M., Effects of fluid properties on hydrodynamic dispersion: comparison of analytical models to numerical simulations, Proc. 1st SPE/IMA Europ. Conf. Mathematics of Oil Recovery, Cambridge, UK, 25-27 July 1989.

[13] Kempers, L.J.T.M., Dispersive mixing in stable and unstable miscible displacements, PhD thesis Delft University of Technology, The Netherlands, 1991.

[14] Kolditz, O., Ratke, R., Diersch, H.J. \& Zielke, W., Coupled groundwater flow and transport: 1. Verification of density variable flow and transport models, Advances in Water Resources, in print, 1998.

[15] Leijnse, T., Three-dimensional modeling of coupled flow and transport in porous media, Ph.D. thesis, University of Notre Dame, Indiana, USA, 1992.

[16] Von Mises, R. \& Friedrichs, K.O., Fluid dynamics, Applied Mathematics Series 5 (1973), Springer, New York, p. 171

[17] Moser, H, Einfluss der Salzkonzentration auf die hydrodynamische Dispersion im porösen Medium, Mitteilung Nr. 128, Technische Universität Berlin (Ph.D. thesis), 1995.

[18] Ogata, A. \& Banks, R.B., A solution of the differential equation of longitudinal dispersion in porous media, U.S. Geological Survey, Professional paper 411-A (1961), Washington.

[19] Oldenburg, C.M. \& Pruess, K., Disperive transport dynamics in a strongly coupled groundwater-brine flow system, Water Resources Research 31(2) (1995), p.p. 289-302.

[20] Pfannkuch, H. O., Contribution á l'étude des déplacements de fluides miscibles dans un milieu poreux, Revue de l'Institut Francais du Pétrole, Vol. 18 (1963), pp. 215-270.

[21] Saffman, P.G., Dispersion due to molecular diffusion and macroscopic mixing in flow through a network of capillaries, J. Fluid Mech. No 27 (1960), p.p. 194-208. 
[22] Saffman, P.G., A theory of dispersion in a porous medium, J. Fluid Mech. No 36 (1959), p.p. 321-349.

[23] Scheidegger, A.E., The physics of flow through porous media, 3rd edn, Univ. of Toronto Press, Toronto, Canada, 1974, p. 306.

[24] Schincariol, R.A. \& Schwartz, F.W., An experimental investigation of variable density flow and mixing in homogeneous and heterogeneous media, Water Resources Research 26 (1990), p.p. 2317-2329.

[25] Taylor, G.I., Dispersion of soluble matter in solvent flowing slowly through a tube, Proc. Roy. Soc. A, No 1137219 (1953), p.p. 186-203.

[26] Taylor, G.I., The dispersion of matter in turbulent flow through a pipe, Proc. Roy. Soc. A. 223 (1954), p.p. 446-468.

[27] Welty, C. \& Gelhar, L.W., Stochastic analysis of the effects of fluid density and viscosity variability on macro dispersion in heterogeneous porous media, Water Resources Research 27 (1991), p.p. 2061-2075.

[28] Wheatcraft, S.E., Carey, A.E., Glass, R.J. \& O'Rourke, J.P., Non-Fickian ionic diffusion across high-concentration gradients, Water Resources Research 31 (1995), p.p. 2213-2218. 